\title{
Caracterización de aleaciones de magnesio mediante microscopía de fuerza atómica con sonda Kelvin de barrido (SKPFM)
}

\author{
Ana Emilse Coy Echeverría \\ $\mathrm{PhD}$ en Ciencia y Tecnología de Materiales, \\ Universidad Complutense de Madrid \\ Docente Tiempo Completo, Investigadora, Escuela de Ingeniería \\ Metalúrgica y Ciencia de Materiales, \\ Universidad Industrial de Santander UIS \\ Bucaramanga, Colombia \\ anaecoy@uis.edu.co \\ Sandra Judith García Vergara \\ PhD en Ciencia e Ingeniería de la Corrosión, \\ Universidad de Manchester \\ Docente Tiempo Completo, Investigadora, Escuela de Ingeniería \\ Metalúrgica, Universidad Industrial de Santander UIS \\ Bucaramanga, Colombia \\ Peter Skeldon \\ Profesor, Universidad de Manchester \\ Manchester, Inglaterra \\ p.skeldon@manchester.ac.uk
}

\begin{abstract}
Resumen-Dos aleaciones de magnesio ZE41 y AZ91D, producidas comercialmente, fueron estudiadas mediante microscopía de fuerza atómica con sonda Kelvin de barrido (SKPFM, siglas en inglés) con el fin de evaluar el papel de sus fases microconstituyentes en interacciones galvánicas potenciales. Los análisis mostraron que las fases microconstituyentes presentes en ambas aleaciones de magnesio exhibieron diferencias de potenciales Volta positivos con relación a la matriz, indicaron un comportamiento catódico. Dicho comportamiento fue corroborado mediante ensayos de inmersión en una solución agresiva de $\mathrm{NaCl}$ al $3.5 \%$ en peso. Este ensayo confirmó que las fases presentes en las aleaciones estudiadas actuaron como sitios efectivos promoviendo el inicio de corrosión localizada. Además, la intensidad del ataque dependió de los valores de potenciales Volta de cada fase, así como de su distribución en la aleación.
\end{abstract}

Palabras clave—Aleaciones de magnesio, SKPFM, Potencial Volta.

Abstract- Two commercially produced magnesium alloys, ZE41 and AZ91D, were studied by scanning Kelvin probe force microscopy (SKPFM) in order to evaluate the role of their micro-constituent phases on potential galvanic interactions. SKPFM measurements showed that these phases exhibited positive Volta potentials differences relative to the matrix, indicating a cathodic behaviour. The influence of these phases on the initiation of corrosion was examined by immersion tests in 3.5 wt. $\% \mathrm{NaCl}$ solution. Immersion testing confirmed that, for both alloys, the micro-constituents phases were

\author{
Fernando Viejo Abrante \\ PhD Ciencia y Tecnología de Materiales, \\ Universidad Complutense de Madrid \\ Docente Tiempo Completo, Investigador, Escuela de Ingeniería \\ Química, Universidad Industrial de Santander UIS \\ Bucaramanga, Colombia \\ fviejo@uis.edu.co \\ George E. Thompson \\ PHD en Desarrollo de Nuevas Aleaciones Ligeras \\ Profesor, Universidad de Manchester \\ Manchester, Inglaterra \\ george.thompson@ manchester.ac.uk \\ A Mº hich \\ PhD en Ciencia y Tecnología de Materiales, \\ Universidad Complutense de Madrid \\ Investigador Asociado, Universidad de Manchester \\ Manchester, Inglaterra
}

effective sites to promote the beginning of localized corrosion. Further, the intensity of the corrosion attack depended on the Volta potential value for each phase, as well as their distribution within the alloy.

Keywords- Magnesium alloys, SKPF, Volta potential.

\section{INTRODUCCIÓN}

En los últimos años las aleaciones de magnesio han venido siendo objetivo especial de estudio. Con un precio similar y con una menor densidad comparada con el aluminio, el magnesio es una atractiva opción para la industria del sector del transporte, donde sus innumerables cualidades pueden ser usadas con la gran ventaja de contribuir al ahorro de energía y reducir de esta forma el impacto ambiental [1]. Como ejemplo, una significativa reducción de peso puede ser alcanzada a través del uso del magnesio en el diseño de automóviles; cada 10 $\mathrm{kg}$ de acero pueden ser reemplazados por $4 \mathrm{~kg}$ de magnesio, esto garantiza un ahorro de $100 \mathrm{~kg}$ de emisiones de gas sobre la vida útil del vehículo.

Aunque una gran variedad de aplicaciones pueden ser visionadas para las aleaciones de magnesio, el uso actual está limitado debido a su baja resistencia a la corrosión en determinados ambientes. Esta susceptibilidad a la corrosión depende 
considerablemente de su microestructura y del carácter anódico o catódico de las fases y/o microcontituyentes presentes en esta [2]-[4].

Con el fin de profundizar en los mecanismos de corrosión de estas aleaciones, diferentes técnicas han sido utilizadas para dar información suplementaria a los resultados obtenidos mediante los métodos electroquímicos clásicos. La microscopía de fuerza atómica con sonda Kelvin de barrido (SKPFM siglas en inglés) ha sido establecida como una técnica poderosa para caracterizar procesos de corrosión asociados con heterogeneidades locales en las superficies pasivas [5]-[7]. Estudios previos han confirmado a esta técnica como una herramienta útil para generar diferencias de potencial Volta, usadas para estimar la nobleza electroquímica de cada fase micro-constituyente con relación a la matriz adyacente [8]-[13]. Además, la alta resolución lateral ofrecida por esta técnica (alrededor de $0,1 \mathrm{~mm}$ ) ha encontrado una aplicación interesante en la determinación de la contribución de las fases micro-constituyentes en la corrosión localizada de aleaciones ligeras [8], [14],[15].

Basado en estas premisas, el objetivo del presente trabajo fue evaluar dos aleaciones comerciales de magnesio, ZE41 y AZ91D mediante SKPFM, complementariamente con microscopía óptica y electrónica de barrido (FEG-SEM), y espectroscopía dispersiva de rayos $X$ (EDS), con el fin de determinar el papel de las fases microconstituyentes en posibles interacciones galvánicas.

\section{PROCEDIMIENTO EXPERIMENTAL}

\section{A. Materiales}

Los materiales objeto de estudio fueron dos aleaciones de magnesio comerciales, ZE41 y AZ91D, sumistradas por la empresa Magnesium Elektron (Inglaterra). La composición nominal de las aleaciones se encuentra en la Tabla I.

TABLA I

Composición nominal de las aleaciones estudiadas

\begin{tabular}{|c|c|c|c|c|c|c|c|}
\hline \multirow{2}{*}{ Aleación } & \multicolumn{7}{|c|}{ Composición (\% en masa) } \\
\cline { 2 - 9 } & $\mathrm{Zn}$ & $\mathrm{Al}$ & $(\mathrm{La}, \mathrm{Ce})$ & $\mathrm{Zr}$ & $\mathrm{Mn}$ & $\mathrm{Fe}$ & $\mathrm{Mg}$ \\
\hline ZE41 & 3,8 & - & 0,95 & 0,6 & 0,15 & $<0,01$ & Bal. \\
\hline AZ91D & 0,68 & 8,8 & - & - & 0,3 & 0,004 & Bal. \\
\hline
\end{tabular}

\section{B. Caracterización microestructural}

Previo a la caracterización microestructural, las aleaciones fueron desbastadas con papel de carburo de silicio hasta una granulometría P1200; posteriormente fueron pulidas a espejo con pasta de diamante de $0,1 \mathrm{~mm}$. Con el fin de revelar su microestructura, las muestras fueron atacadas con Picral (10 mL de ácido acético, 4,2g de ácido pícrico, $10 \mathrm{~mL}$ de $\mathrm{H} 20$ y $70 \mathrm{~mL}$ de etanol). La observación de las muestras fue llevada a cabo por microscopía óptica (OM), mediante un microscopio Olympus GX71, y por microscopía electrónica de barrido (SEM), con un microscopio Philips XL30 equipado con detectores para análisis mediante espectroscopía de energía dispersiva de rayos $X$ (EDS) y electrones retrodispersados (EB).

\section{Estudio mediante microscopía de fuerza ató- mica con sonda Kelvin de barrido- SKPFM}

SKPFM fue usado para determinar las diferencias de potencial Volta entre las fases microconstitueyentes y la matriz. Estos datos pueden ser usados para estimar la nobleza de cada microconstituyente con relación a la matriz y de esta forma determinar las potenciales interacciones galvánicas que pueden generar el inicio de un ataque por corrosión.

Antes de realizar este estudio, las muestras fueron pulidas a espejo con pasta de diamante de $0,1 \mathrm{~mm}$, con una solución de etilenglicol como lubricante y posteriormente éstas fueron limpiadas con etanol en un baño ultrasónico. Las medidas mediante SKPFM fueron realizadas inmediatamente después de la limpieza.

El microscopio de fuerza atómica empleado fue un Nanoscope DimensionTM 3100 operado en una secuencia de tapping/lift. Este instrumento puede reproducir la topografía superficial y medir la distribución de potenciales Volta simultáneamente mediante puntas eléctricamente conductoras. Para el presente trabajo se utilizó una punta de silicio con recubrimiento de platino de $20 \mathrm{~nm}$ de espesor. Todas las medidas fueron realizadas a temperatura ambiente y con una humedad relativa controlada entre 40 y $65 \%$.

\section{Ensayos de inmersión}

Con el fin de confirmar los sitios preferenciales para el inicio del proceso de corrosión, las mues- 
tras fueron sumergidas en una solución de $\mathrm{NaCl}$ al 3,5\% en peso, para tiempos que fueron desde 5 hasta $60 \mathrm{~min}$. Finalmente, la morfología de corrosión, después de los ensayos de inmersión, fue evaluada mediante SEM.

\section{RESULTADOS Y DISCUSIÓN}

\section{A. Caracterización microestructural}

Las Fig. 1a) y 1b) muestran las micrografías correspondientes a la aleación ZE41 tomadas mediante OM y SEM, respectivamente. La microestructuctura de esta aleación consistió de dendritas de $\alpha-\mathrm{Mg}$ (matriz) rodeadas por una fase eutéctica y pequeños precipitados dispersos de forma aleatoria dentro de la aleación, ver [Fig. 1a].

El eutéctico correspondió a la fase T (Mg7Zn3RE), donde RE incluye los elementos Ce y La. Con respecto a los pequeños precipitados un análisis SEM/EDS reveló que, en general, los precipitados estuvieron enriquecidos en $\mathrm{Zr}$ y $\mathrm{Zn}$, con pequeñas cantidades de Fe, ver [Fig. 1b)].

Con respecto a la aleación AZ91D, la microestructura consistió de una matriz de dendritas primarias de a- $\alpha \mathrm{Mg}$ y un eutéctico parcialmente divorciado $\alpha-\mathrm{Mg} /$ fase $ß-\mathrm{Mg} 17 \mathrm{Al} 12$ en la región interdendrítica, ver [Fig. 2a)], finalmente, inclusiones intermetálicas de Al-Mn fueron también encontradas, principalmente Al-Mn, ver [Fig. 2b)].

FIG. 1. CARACTERIZACIÓN MICROESTRUCTURAL DE LA ALEACIÓN ZE41. A) MICROGRAFÍA TOMADA MEDIANTE MICROSCOPIA ÓPTICA, B) MICROGRA-

FÍA SEM TOMADA MEDIANTE ELECTRONES RETRODISPERSADOS
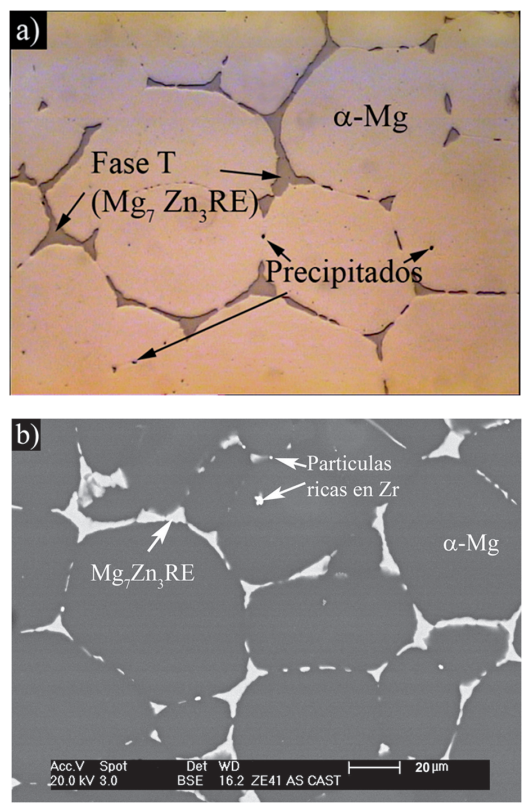

\section{B. Estudio mediante microscopía de fuerza ató- mica con sonda Kelvin de barrido- SKPFM}

Los mapas de potencial obtenidos mediante SKPFM sobre un área seleccionada de las aleaciones objeto de estudio se muestran en las Fig 3a y 3b. Los mapas revelan las diferencias de potencial Volta que existen entre la matriz y las fases microconstituyentes. Los colores claros representan las áreas catódicas, mientras que los colores oscuros representan las áreas anódicas.

En la aleación ZE41, la fase T (Mg7Zn3RE) presentó una diferencia de potencial Volta alrededor de $+100 \mathrm{mV}$ con respecto a la matriz adyacente, mientras que los pequeños precipitados enriquecidos en Zr y Zn presentaron las más altas diferencias, alrededor de $+180 \mathrm{mV}$ con respecto al área adyacente, ver [Fig. 2a]. De esta forma, estos dos tipos de precipitados pueden actuar como potenciales cátodos en un proceso de corrosión microgalvánica, que favorece el inicio del ataque corrosivo en las áreas adyacentes a éstos. Por otro lado, los límites de grano aparecen definidos claramente como las zonas más oscuras, que representan así áreas anódicas, ver [Fig. 2a]. Un análisis previo realizado mediante EDS reveló un empobrecimiento de elementos aleantes en estas zonas, esto pudo ser la causa de su respuesta anódica.

FIG. 2. CARACTERIZACIÓN MICROESTRUCTURAL DE LA ALEACIÓN AZ91D. A) MICROGRAFÍA TOMADA MEDIANTE MICROSCOPÍA ÓPTICA, B) MICROGRAFÍA SEM TOMADA MEDIANTE ELECTRONES RETRODISPERSADOS
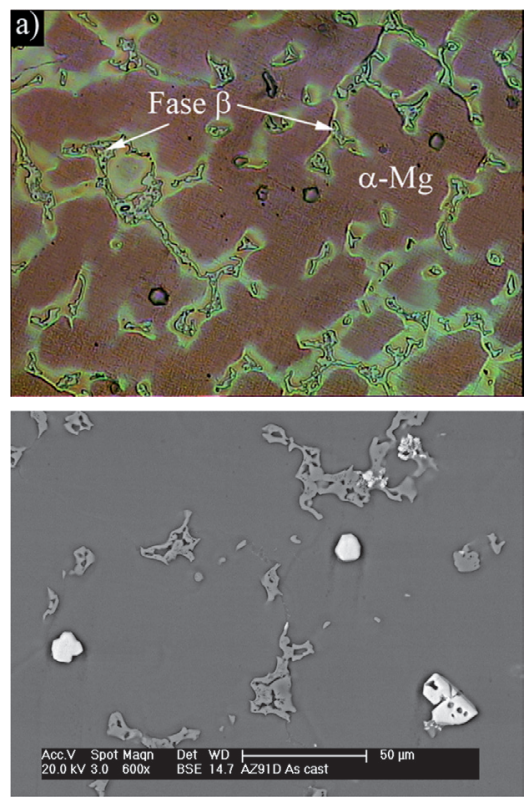
El mapa de potencial Volta obtenido de un área seleccionada de la aleación AZ91D mostró que, los precipitados intermetálicos presentaron las más altas diferencias de potencial Volta (alrededor de $+300 \mathrm{mV}$ ) con respecto a la matriz adyacente, mientras que la fase $b$ exhibió diferencias alrededor de $+50 \mathrm{mV}$. Estas diferencias fueron insignificantes comparadas con los valores mostrados por los precipitados intermetálicos, los cuales pueden actuar como cátodos potenciales durante un proceso de corrosión microgalvánica.

FIG. 3. MAPAS DE POTENCIAL VOLTA OBTENIDOS MEDIANTE SKPFM SOBRE UN ÁREA SELECCIONADA DE LA ALEACIÓN. A) ZE41, B) AZ91D
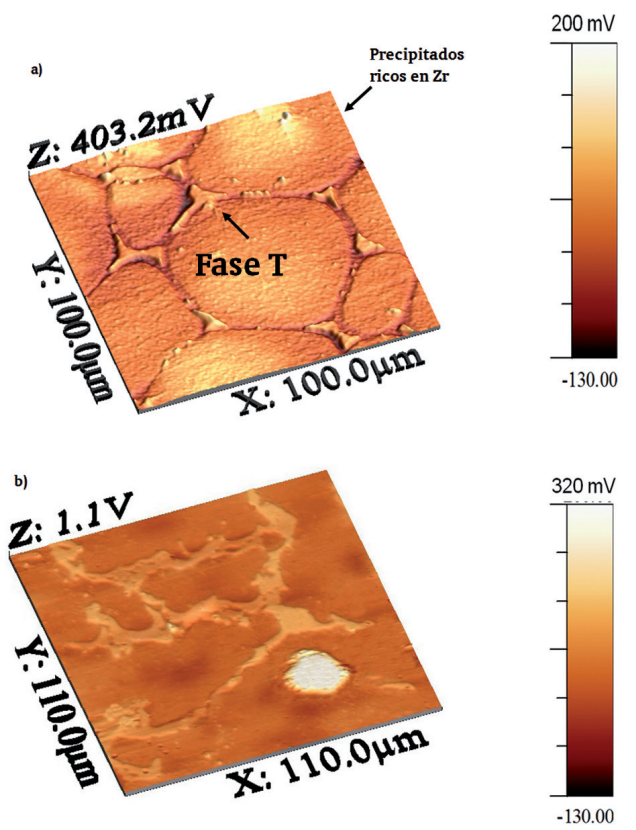

Como era de esperarse, en el caso de la aleación ZE41, el ataque localizado comenzó con la disolución de los límites de grano. Este proceso fue intensificado por la presencia de la fase interdendrítica T, ver [Fig. 4a)], la cual actúa como cátodo de acuerdo con el estudio realizado mediante SKPFM. Como resultado, la matriz $\alpha-\mathrm{Mg}$ adyacente sufrió corrosión, mientras que la fase intendendrítica permaneció inalterada.

Con respecto a la aleación AZ91D, el ataque inició en las áreas adyacentes a las partículas intermetálicas Al-Mn, mientras que en la interface matriz-fase $ß$ no se observó un ataque localizado, ver [Fig. 4b)]. Este comportamiento fue debido al carácter fuertemente catódico de las partículas intermetálicas, comparado con la fase $ß$ y que fue determinado mediante SKPFM.

\section{Ensayos de inmersión}

Las Fig. 4a y 4b muestran las micrografías tomadas mediante SEM de las superficies corroídas de las aleaciones ZE41 y AZ91D, respectivamente, después de la inmersión en una solución de $\mathrm{NaCl}$ al $3.5 \%$ en peso, durante $5 \mathrm{~min}$.

FIG. 4. MICROGRAFÍAS SEM DE LAS SUPERFICIES ATACADAS DESPUÉS DE SU INMERSIÓN EN LA SOLUCIÓN DE NACL AL 3,5\% DURANTE 5 MIN. A) ZE41 B) AZ91 D
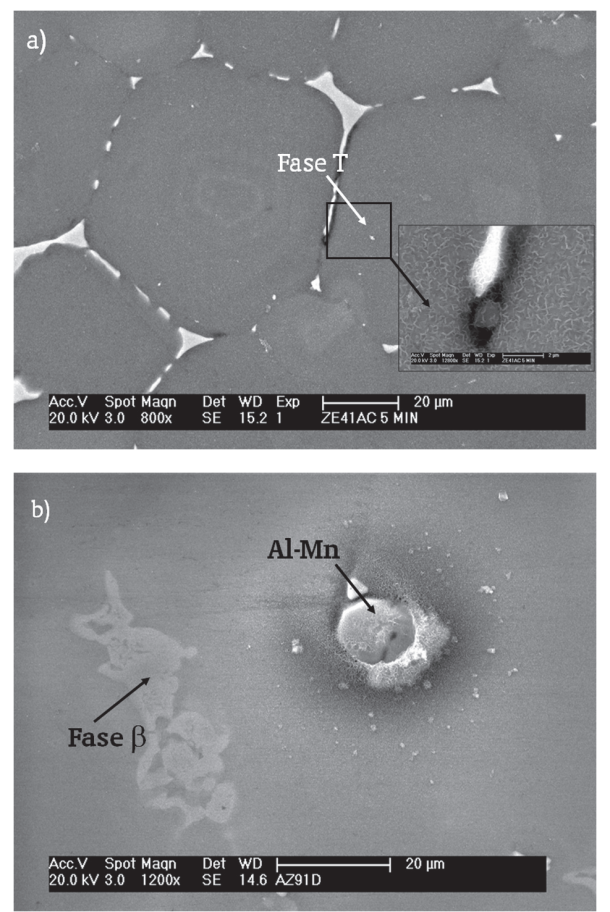

\section{CONCLUSIONES}

Se confirmó que la SKPFM es una herramienta poderosa para evaluar el papel galvánico de los microconstituyentes presentes en las aleaciones de magnesio. Así, la intensidad de la corrosión microgalvánica dependió de las diferencias de potencial Volta de cada microcontituyente, aunque la extensión del ataque dependió del tamaño y la distribución de los mismos.

La aleación ZE41 presentó un fuerte ataque micro-galvánico debido al efecto combinado de la fase catódica T (Mg7Zn3RE) y el empobrecimiento de las áreas anódicas, ambas presentes en el límite de grano.

En la aleación AZ91D, las fases con el más alto carácter catódico correspondieron a los precipitados intermetálicos Al-Mn, evidenciándose corrosión en sus áreas adyacentes. Por otro lado, los bajos valores de diferencia de potencial Volta de 
la fase b en relación con la matriz no señalaron un inició de ataque preferencial en sus áreas adyacentes para los tiempos estudiados.

\section{AGRADECIMIENTOS}

Agradecimientos al Corrosion and Protection Centre de University of Manchester (Reino Unido, EP/D029201/1) por su inestimable aporte técnico y científico que permitió la realización de este trabajo y a la Vicerrectoría de Investigación y Extensión de la Universidad Industrial de Santander por la financiación del mismo (Desarrollo de Materiales y Recubrimientos de Interés Tecnológico, Código 5450).

\section{REFERENCIAS}

[1] I.J. Polmear, Light Alloys - from traditional alloys to nanocrystals, 4Th ed., Edward Arnold, Sevenoaks, UK, 2005

[2] A. Pardo, M.C. Merino, A.E. Coy, R. Arrabal, F. Viejo, E. Matykina, Corros. Sci. 50 (2008). pp 823834

[3] M.Ch. Zhao, M. Liu, G. Song, A. Atrens. Corros. Sci. 50 (2008) 1939-1953

[4] A. Pardo, M.C. Merino, A.E. Coy, F. Viejo, R. Arrabal, S. Feliú Jr. Electrochimica Acta, 53 (2008). pp 7890-7902

[5] V. Guillaumin. P. Schmutz, G. S. Frankel, J. Electrochem. Soc. 148 (2001). pp B163-B173

[6] F. Andreatta, H. Terryn, Corros. Sci. 45 (2003). Pp 1733-1746

[7] M. Femenia, C. Canalias, J. Pan, J. Leygraf, J. Electrochem. Soc. 150 (2003). pp B274-B281

[8] D. B. Blücher. J.E. Svensson. L.G. Johansson. M. Rohwerder, M. Stratmann, J. Electrochem. Soc. 151 (2004). pp B621-B626

[9] M. Jönsson, D. Thierry, N. LeBozec, Corros. Sci. 48 (2006) pp 1193-1208

[10] F. Andreatta, I. Apachitei, A. A. Kodentsov, J. Dzwonczyk, J. Duszczyk, 51 (2006) pp 35513557

[11] M. Jönsson, D. Persson, R. Gubner, J. Electrochem. Soc.154 (2007) pp C684-C691
[12] I. Apachitei, L.E. Fratila-Apachitei, J. Duszczyk, 57 (2007) pp 1012-1015

[13] S. Rahman, G. Priyadarshan, K.S. Raja, C. Nesbitt, M. Misra, Mater. Lett. 62 (2008). pp 22632266

[14] P. Schmutz, G.S. Frankel, J. Electrochem. Soc. 145 (1998). pp 2285-2295

[15] A.E. Coy, F. Viejo, P. Skeldon, G.E. Thompson. Corros. Sci. 52, (2010). pp 3896-3906 